\title{
Reproduction et croissance de deux races de bovins (N'Dama et croisées) dans le district des Montagnes à l'ouest de la Côte d'Ivoire
}

\author{
Nicolas Bosson Aboly ${ }^{1 *}$ Mathurin Koffi Konan ${ }^{1}$ \\ Gatien Konan Gboko Brou ${ }^{1}$ Nahoulé Silué ${ }^{1}$ René Yadé Soro ${ }^{2}$
}

\section{Mots-clés}

Bovin, Montbéliarde, N’Dama, produit de croisement, reproduction animale, performance animale, Côte d'Ivoire

Submitted: 15 August 2020

Accepted: 4 February 2021

Published: 30 June 2021

DOI: $10.19182 /$ remvt.36366

\section{Résumé}

L'étude a été menée à l'ouest de la Côte d'Ivoire dans le district des Montagnes sur 120 vaches et 96 veaux mâles et femelles de races N'Dama et croisées (Montbéliarde x N'Dama) pendant 36 mois afin de déterminer leurs performances de reproduction et de croissance. Les résultats ont révélé que le taux de gravidité et la durée de lactation des vaches croisées (demi-sang Montbéliardes) étaient plus élevés que ceux des N'Dama. De même, l'intervalle entre vêlages des vaches croisées (428 $\pm 16,6$ jours) a été inférieur à celui des N'Dama (452 $\pm 12,8$ jours). Le poids à la naissance des veaux croisés (23 à $25 \mathrm{~kg}$ ) a été supérieur à celui des N’Dama (17 à $19 \mathrm{~kg}$ ). Le gain moyen quotidien sur toute la période a été de 356,8 $\pm 47,7$ g pour les mâles et de $324,8 \pm 47,1 \mathrm{~g}$ pour les femelles chez les croisés, contre respectivement $291,7 \pm 57,6$ g et 266,6 $249,1 \mathrm{~g}$ chez les N'Dama. Enfin, le poids adulte a été de $370 \pm 1,7 \mathrm{~kg}$ pour les mâles et $300 \pm 2,3 \mathrm{~kg}$ pour les femelles chez les croisés, contre respectivement $235 \pm 1,6 \mathrm{~kg}$ et $230 \pm 2,1 \mathrm{~kg}$ chez les N'Dama. Les performances de reproduction et de croissance des croisés ont été significativement plus élevées $(p<0,05)$ que celles des N’Dama.

- Comment citer cet article : Aboly N.B., Konan M.K., Brou G.K.G., Silué N., Soro R.Y., 2021. Reproduction and growth of two breeds of cattle (N'Dama and crossbred) in the Mountains' District of Western Côte d'Ivoire. Rev. Elev. Med. Vet. Pays Trop., 74 (2): 115-120, doi: 10.19182/remvt.36366

\section{INTRODUCTION}

L'agriculture et l'élevage constituent les principales sources de revenus des populations rurales en Afrique (Metzger et al., 1995). En Côte d'Ivoire, ces secteurs emploient les deux tiers de la population active et contribuent au produit intérieur brut pour $34 \%$ et aux recettes d'exportation pour $66 \%$ (Akaffou, 2013). Cependant, le système de production des races locales ne permet pas de satisfaire la demande des populations en protéines animales, d'où une situation

\footnotetext{
1. Institut de gestion agropastorale (IGA), Université Péléforo Gon Coulibaly de Korhogo, Laboratoire de production et santé animale, BP 1223, Korhogo, Côte d'Ivoire.

2. Laboratoire de biochimie, Université Félix Houphouet-Boigny d'Abidjan-Cocody / UFR Biosciences, Abidjan 22, Côte d'Ivoire.

* Auteur pour la correspondance

Tél. : +22507567160 / 02918398 ; email : abolyb@upgc.edu.ci
}

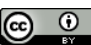

https://creativecommons.org/licenses/by/4.0/ de dépendance vis-à-vis de l'extérieur en matière d'approvisionnement en produits carnés et laitiers (Atsé, 1990). Avec une population estimée à 23 millions d'habitants en 2016 et un taux de croissance moyen annuel de $3,8 \%$, les importations en protéines animales pour satisfaire les besoins de consommation de la population ne cessent pas (Coulibaly, 2013 ; Mirah, 2014). Face à ce handicap majeur de l'économie, l'Etat ivoirien a opté pour le développement de l'élevage bovin. En effet, l'une des alternatives permettant l'augmentation sensible de la production locale est l'amélioration du potentiel génétique des races locales par le biais du croisement de bovins de races locales avec des races exotiques (Koné, 2010). Aujourd'hui, la race amélioratrice utilisée est la Montbéliarde et les animaux à améliorer sont les vaches de race N'Dama trypanotolérantes. Vu l'intérêt que suscitent les croisées (Montbéliarde x N'Dama), il est apparu nécessaire d'effectuer une étude sur ce matériel biologique qui demeure encore peu connu du point de vue de ses performances zootechniques. L'objectif de l'étude a été de déterminer les performances des races N'Dama et croisées en termes de reproduction et de croissance. 


\section{MATERIEL ET METHODES}

\section{Milieu d'étude}

Létude a été menée dans le district des Montagnes dans l'ouest de la Côte d'Ivoire, qui compte une population de 2371920 habitants en majorité rurale et à vocation agricole (INS, 2014). Le climat de montagne y est de type équatorial caractérisé par deux saisons sèches (une grande saison sèche de novembre à mars et une petite saison sèche de juillet à fin août) et deux saisons de pluie (une grande saison de pluie de mars à juin et une petite saison de pluie de septembre à octobre) (Mirah, 2014).

\section{Caractéristiques des élevages sélectionnés}

L'étude a pris en compte les données de reproduction des vaches et de croissance des veaux mâles et femelles couvrant la période de mars 2016 à février 2019, soit 36 mois. La méthodologie a consisté à faire un choix simple et aléatoire des fermes sur la base de la disponibilité des éleveurs, de la présence dans le troupeau de 20 femelles reproductrices au moins, de l'existence dans les fermes d'un parc de nuit, d'un abreuvoir, d'une mangeoire et si possible d'un couloir de contention. Les critères dans le choix des élevages ont été leur importance, l'utilisation de l'insémination artificielle et dans une moindre mesure l'accessibilité. Le matériel biologique était constitué de 240 vaches N'Dama, 120 vaches croisées de différents âges et 288 veaux nés dans les élevages retenus.

\section{Conduite des animaux}

Lalimentation de base des animaux était le pâturage naturel dominé par Andropogon gayanus. Toutefois, le pâturage artificiel d'appoint de Panicum maximum $\mathrm{C} 1$ a été mis en place. Le temps de pâture était de 8 à 10 heures par jour. A la mi-journée, les animaux revenaient à l'étable pour s'abreuver durant une heure. Les vaches lactantes et les veaux recevaient des compléments alimentaires composés de son de maïs ou de maïs concassé, de tourteau de coprah, de graines de coton, de minéraux, et de résidus de récoltes issus des champs de cultures.

Chaque vache recevait comme quantité de concentré le poids équivalent à la moitié de sa production laitière de la veille (1 à 3,5 kg). Tous les animaux avaient un accès libre à la pierre à lécher (sel + minéraux). Pour minimiser les facteurs de variation pouvant influencer les performances de reproduction et de croissance surtout des croisés au niveau de la trypanosomose et des ectoparasitoses (tiques), un plan de prophylaxie a été appliqué.

Pour le suivi sanitaire des animaux, en plus des campagnes de vaccination obligatoire (péripneumonie contagieuse bovine, pasteurellose et charbon symptomatique), les vaches et les veaux ont été traités contre les parasites gastro-intestinaux et externes à l'aide respectivement d'albendazole 300 ( 1 bolus pour $60 \mathrm{~kg}$ de poids vif), de lévamisole (7,5 ml pour $200 \mathrm{~kg}$ de poids vif, Levasol $20 \%$ Oral) et de cyperméthrine (1 $\mathrm{ml} / \mathrm{L}$ d'eau, Dominex).

\section{Animaux}

Les données ont été collectées dans 18 élevages bovins dont 12 de N'Dama et six de croisés. Vingt vaches et seize veaux (huit mâles et huit femelles) ont été retenus par élevage de chaque race pendant une période de lactation. Toutes les données relatives à la reproduction ont été obtenues à partir de documents de suivi du cheptel qui ont été fournis par les éleveurs.

Les vaches locales N'Dama ont été choisies par les éleveurs parce qu'elles sont rustiques et trypanotolérantes. Les vaches retenues pour l'insémination artificielle en étaient à leur deuxième vêlage et étaient âgées de quatre ou cinq ans. Les veaux et les vaches croisés étaient de type génétique F1 (Montbéliards x N'Dama). Ces vaches ont servi de matrices d'insémination artificielle avec la semence de taureaux de races Montbéliarde (vaches N'Dama) et N'Dama (vaches F1), soit 288 veaux au total. Les animaux choisis dans les élevages ont été identifiés par des boucles d'oreille numérotées.

\section{Paramètres mesurés ou calculés}

L'insémination artificielle a été la méthode de reproduction choisie pour l'étude. Elle a été réalisée à partir des programmes d'insémination basés sur la synchronisation des chaleurs de plusieurs vaches. Cependant, les éleveurs ont souvent eu recours à la monte naturelle quand il y avait une rupture de stocks de produits d'insémination.

D'autres mesures ont porté sur les paramètres suivants : l'âge au premier vêlage (âge d'une femelle à la naissance de son premier veau) ; l'intervalle entre vêlages (nombre de jours s'écoulant entre deux vêlages consécutifs d'une même femelle) ; la durée de lactation (nombre de jours pendant lesquels la femelle qui a mis bas produit du lait) ; la production laitière par vache (quantité totale de lait produit par une vache pendant la durée de lactation); et les poids vifs des veaux enregistrés à la naissance et aux âges de 3, 6, 12, 18, 24 et 36 mois, soit sept pesées. Une balance de type bascule PM 41 d'une portée de $500 \mathrm{~kg}$ et de précision $0,02 \%$ a été utilisée.

Concernant le gain de poids moyen quotidien (GMQ), le mode de calcul a été le suivant : pour un animal donné, nous disposions d'un résultat de pesée $P_{1}$ pour un âge de $x$ jours, ainsi que d'un résultat de pesée $P_{2}$, effectuée $y$ jours plus tard, alors que l'animal avait $(x+y)$ jours. Soit GMQ ${ }_{1 / 2}$, le gain moyen de croissance quotidienne entre les deux pesées dont le calcul a été réalisé selon la formule : GMQ $_{1 / 2}=\left(P_{2}-P_{1}\right) / y$. Ainsi, huit classes d'âge ont été définies sur la période de calcul du GMQ : naissance-3 mois ; 3-6 mois ; 6-9 mois ; 9-12 mois ; 12-18 mois ; 18-24 mois ; 24-30 mois et $30-36$ mois.

La quantité de lait par vache a été mesurée chaque jour à partir de pesées quotidiennes des veaux qui ont été mis à jeun pendant 12 heures. Ceux-ci ont été pesés avant et après avoir tété presque tout le lait de leur mère.

\section{Analyses statistiques}

L'analyse statistique des données a été effectuée avec les logiciels Excel version 2013 et XLSTAT 2020 version 5.1. Les comparaisons des paramètres de reproduction entre les vaches N'Dama, et Montbéliardes x N'Dama, d'une part, et de croissance entre les veaux mâles et femelles, d'autre part, ont été réalisées en utilisant le Test $\mathrm{U}$ de Mann-Whitney au seuil de $5 \%$.

\section{RESULTATS}

\section{Paramètres de reproduction des vaches N'Dama et croisées}

Les performances de reproduction des vaches N'Dama et croisées sont présentées dans le tableau I. Les taux de gravidité et de vêlage des vaches croisées ont été supérieurs respectivement de $8 \%$ et $12 \%$ à ceux des N'Dama. La durée moyenne de lactation des N'Dama a été plus courte de 60 jours que celle des croisées. En revanche, l'intervalle moyen entre vêlages des vaches N'Dama a été plus long de 24 jours que celui des croisées. Les productions laitières moyennes par vache ont été de 1,3 $\pm 0,2$ litre chez les N'Dama, et de 7,1 $\pm 0,2$ litres chez les vaches croisées. L'analyse de ces paramètres a mis en évidence une différence significative entre les races de vaches $(\mathrm{p}<0,05)$, à l'exception de l'intervalle moyen entre vêlages. 
Tableau I : Paramètres de reproduction des vaches N'Dama et croisées en Côte d'Ivoire /// Reproduction parameters of N'Dama and crossbred cows in Côte d'Ivoire

\begin{tabular}{|c|c|c|c|}
\hline $\begin{array}{l}\text { Paramètre de } \\
\text { reproduction }\end{array}$ & $\begin{array}{l}\text { N'Dama } \\
(n=240) \\
\text { Moy. } \pm \text { ET } \\
(m i n-m a x)\end{array}$ & $\begin{array}{l}\text { N'Dama } x \\
\text { Montbéliard } \\
(n=120) \\
\text { Moy. } \pm \text { ET } \\
\text { (min-max) }\end{array}$ & $\mathbf{P}$ \\
\hline $\begin{array}{l}\text { Taux de gravidité } \\
(\%)\end{array}$ & $\begin{array}{l}79 \pm 4,3 \\
(75-86,6)\end{array}$ & $\begin{array}{c}87,2 \pm 9,2 \\
(71,4-94,7)\end{array}$ & 0,031 \\
\hline Taux de vêlage ( \%) & $\begin{array}{c}60 \pm 3,64 \\
(53,8-66,1)\end{array}$ & $\begin{array}{l}72,5 \pm 3,64 \\
(50-90)\end{array}$ & 0,057 \\
\hline $\begin{array}{l}\text { Durée de lactation } \\
\text { (jours) }\end{array}$ & $\begin{array}{l}176 \pm 2,1 \\
(173-179)\end{array}$ & $\begin{array}{l}235 \pm 3,3 \\
(230-241)\end{array}$ & 0,005 \\
\hline $\begin{array}{l}\text { Age au premier } \\
\text { vêlage (mois) }\end{array}$ & $\begin{array}{l}36 \pm 0,5 \\
(35-37)\end{array}$ & $\begin{array}{c}38 \pm 3 \\
(36-39)\end{array}$ & 0,7 \\
\hline $\begin{array}{l}\text { Intervalles entre } \\
\text { vêlages (jours) }\end{array}$ & $\begin{array}{l}452 \pm 12,8 \\
(428-473)\end{array}$ & $\begin{array}{l}428 \pm 16,6 \\
(398-482)\end{array}$ & 0,7 \\
\hline $\begin{array}{l}\text { Production } \\
\text { moyenne de lait / } \\
\text { jour/vache (L) }\end{array}$ & $\begin{array}{c}1,3 \pm 0,2 \\
(1-1,8)\end{array}$ & $\begin{array}{l}7,1 \pm 0,2 \\
(6,5-7,5)\end{array}$ & 0,005 \\
\hline $\begin{array}{l}\text { Production laitière / } \\
\text { vache/lactation (L) }\end{array}$ & $\begin{array}{c}236 \pm 27,3 \\
(201,4-279,8)\end{array}$ & $\begin{array}{c}1607,5 \pm 145,5 \\
(1312,6-1692,1)\end{array}$ & 0,002 \\
\hline
\end{tabular}

Moy. \pm ET (min-max) : moyenne \pm écart-type (minimum-maximum) /// Moy. \pm ET (min-max): mean \pm standard error (minimum-maximum)

\section{Performances pondérales des veaux N'Dama et croisés (F1 et un-quart-de-sang Montbéliards)}

Les performances pondérales des veaux N'Dama et des croisés (F1 et un-quart-de-sang Montbéliards) sont présentées dans le tableau II. Le poids moyen à la naissance des veaux a été différent en fonction du sexe et de la race de bovins. Les veaux croisés étaient des F1 (demisang Montbéliards), et des rétrocroisé (un-quart-de-sang Montbéliards). Leurs poids vifs ont été supérieurs à ceux des N'Dama de la naissance à 36 mois d'âge, sauf pour les femelles âgées de six mois. Néanmoins, à trois mois, les velles N'Dama et les croisées F1 ont atteint le même poids vif $(50 \mathrm{~kg})$, supérieur de $10 \mathrm{~kg}$ à celui des velles rétrocroisées. Il faut noter que les poids vifs des mâles ont été supérieurs ou égaux à ceux des femelles de la naissance à six mois. En revanche à 12 mois, les velles croisées F1 ont pesé en moyenne $5 \mathrm{~kg}$ de plus que les veaux croisés F1. A ce même âge, les mâles N'Dama ne pesaient en moyenne que $140 \pm 1,3 \mathrm{~kg}$ et les femelles $125 \pm 0,6 \mathrm{~kg}$ (tableau II).

Le poids adulte moyen des croisés $\mathrm{F} 1$ de $370 \pm 1,7 \mathrm{~kg}$ pour les mâles et $300 \pm 2,4 \mathrm{~kg}$ pour les femelles ont été supérieurs à ceux des rétrocroisés avec $300 \pm 0,5 \mathrm{~kg}$ pour les mâles et $260 \pm 0,1 \mathrm{~kg}$ pour les femelles, eux-mêmes plus lourds que les N'Dama avec seulement $235 \mathrm{~kg} \pm 1,6 \mathrm{~kg}$ pour les mâles et $230 \pm 2,1 \mathrm{~kg}$ pour les femelles. L'analyse des paramètres de poids a mis en évidence une différence significative entre les veaux croisés et N'Dama $(p<0,05)$.

\section{Croissance des veaux N'Dama, croisés F1 et rétrocroisés}

Les GMQ des veaux N'Dama et croisés F1 (demi-sang Montbéliards) et rétrocroisés (un-quart-de-sang Montbéliards) sont présentés dans le tableau III. La croissance est estimée par le calcul du gain moyen quotidien (GMQ) depuis la naissance et sur une période de 36 mois. Les GMQ des veaux et des velles ont été inférieurs à $500 \mathrm{~g}$ sur la période d'étude. Toutefois, dès la naissance et sur toute la période, les mâles croisés F1 ont eu des GMQ supérieurs à ceux des mâles rétrocroisés et N'Dama (figure 1).

Les GMQ entre les âges types de 6,12 et 18 mois ont été plus faibles que ceux de la phase lactée de 0 à 3 mois. Une chute drastique du GMQ a été observée entre les périodes de 0 à 3 mois chez les femelles et de 3 à 6 mois chez les mâles avant de croître.

Dans l'ensemble le GMQ est demeuré inférieur chez les N'Dama comparés aux croisés $\mathrm{F} 1$ et rétrocroisés de la naissance à l'âge adulte. Cependant, l'évolution du GMQ des velles à partir de 6 mois s'est faite en dents de scie (figure 2). Les GMQ des veaux mâles et femelles N'Dama n'ont pas été significativement différents $(p>0,05)$ de ceux des croisés.

\section{DISCUSSION}

L'étude s'est intéressée aux performances de reproduction et de croissance aussi bien des vaches et veaux N'Dama que des croisés (un-demi, et un-quart-de-sang Montbéliards). Le taux de gravidité et de vêlage des N'Dama a été inférieur respectivement de $8 \%$ et $12 \%$ à celui des demi-sang Montbéliards. Cela pourrait s'expliquer par le fait que le phénomène d'hétérosis sur le caractère de fertilité

Tableau II : Performance pondérale $(\mathrm{kg})$ des veaux N'Dama et croisés en Côte d'Ivoire /// Weight performance ( $\mathrm{kg}$ ) of $\mathrm{N}^{\prime} \mathrm{Dama}$ and crossbred calves in Côte d'Ivoire

\begin{tabular}{lccc} 
Age & $\begin{array}{c}\text { Veaux métis F1 }(\mathbf{n}=\mathbf{9 6}) \\
\text { Moy. } \pm \text { ET (min-max) }\end{array}$ & $\begin{array}{c}\text { Veaux N'Dama }(\mathbf{n}=96) \\
\text { Moy. } \pm \text { ET }(\mathbf{m i n}-\mathbf{m a x})\end{array}$ & $\begin{array}{c}\text { Veaux métis rétrocroisés }(\mathbf{n}=\mathbf{9 6}) * \\
\text { Moy. } \pm \text { ET }(\mathbf{m i n}-\mathbf{m a x})\end{array}$ \\
\hline Naissance (mâle) & $25 \pm 0,2(24,7-25,3)$ & $19 \pm 0,2(18,6-19,4)$ & $20 \pm 0,2(19-21)$ \\
Naissance (femelle) & $23 \pm 0,4(22-23)$ & $17 \pm 0,1(17,2-18,6)$ & $18 \pm 0,3(17-20)$ \\
3 mois (mâle) & $70 \pm 0,7(68,7-71)$ & $55 \pm 0,6(53-56)$ & $40 \pm 2,1(39-42)$ \\
3 mois (femelle) & $50,5 \pm 1,5(51-53)$ & $50 \pm 1,6(48-52)$ & $40 \pm 2(38-43)$ \\
6 mois (mâle) & $95 \pm 0,5(94-96)$ & $85 \pm 1,3(83-87)$ & $80 \pm 1,7(70-82)$ \\
6 mois (femelle) & $95 \pm 0,1(94,7-95)$ & $75 \pm 0,6(75-85)$ & $65 \pm 2,3(63-67)$ \\
9 mois (mâle) & $125 \pm 2(123-127)$ & $110 \pm 1,6(108-113)$ & $115 \pm 2,9(110-118)$ \\
9 mois (femelle) & $120 \pm \pm 1,4(117-122)$ & $90 \pm 1(88-92)$ & $108 \pm 1,7(105-110)$ \\
12 mois (mâle) & $160 \pm 1,4(155-162)$ & $140 \pm 1,3(138-142)$ & $135 \pm 0,8(150-165)$ \\
12 mois (femelle) & $165 \pm 1,3(163-165)$ & $125 \pm 0,6(124-126)$ & $300 \pm 0,3(128-132)$ \\
Adulte mâle & $370 \pm 1,7(368-372)$ & $235 \pm 1,6(235-235)$ & $260 \pm 0,1(255-265)$
\end{tabular}

* N'Dama et croisés F1, p =0,516; N'Dama et rétrocroisés, $\mathrm{p}=0,954 / / /$ * N'Dama and F1 crossbred, $p=0.516$; N'Dama and backcrossed, $p=0.954$ 
Tableau III : Gain de poids moyen quotidien des veaux N'Dama et croisés de la naissance à 36 mois d'âge en Côte d'Ivoire /// Daily weight gain from birth to 36 months of N'Dama and crossbred calves in Côte d'Ivoire

\begin{tabular}{|c|c|c|c|c|c|c|}
\hline \multirow[t]{2}{*}{$\begin{array}{l}\text { Age } \\
\text { (mois) }\end{array}$} & \multicolumn{2}{|c|}{$\begin{array}{c}\text { Métis F1 ( } n=96) \\
\text { Moy. } \pm \text { ET (min-max) }\end{array}$} & \multicolumn{2}{|c|}{$\begin{array}{c}\text { N'Dama } \mathbf{n}=96) \\
\text { Moy. } \pm \text { ET (min-max) }\end{array}$} & \multicolumn{2}{|c|}{$\begin{array}{l}\text { Métis rétrocroisés } \mathrm{n}=96) \\
\text { Moy. } \pm \text { ET (min-max) }\end{array}$} \\
\hline & Mâle & Femelle & Mâle & Femelle & Mâle & Femelle \\
\hline 3 & $\begin{array}{c}487,3 \pm 0,6 \\
(485-489)\end{array}$ & $\begin{array}{r}331,5 \pm 5,7 \\
(329-333)\end{array}$ & $\begin{array}{r}388,7 \pm 5,3 \\
(387-392)\end{array}$ & $\begin{array}{r}356,9 \pm 1,4 \\
(352-358)\end{array}$ & $\begin{array}{r}388,7 \pm 5,3 \\
(386-389)\end{array}$ & $\begin{array}{r}356,9 \pm 1,4 \\
(354-357)\end{array}$ \\
\hline 6 & $\begin{array}{r}381,3 \pm 0,5 \\
(380-383)\end{array}$ & $\begin{array}{c}391,3 \pm 1,6 \\
(389-393)\end{array}$ & $\begin{array}{c}360,6 \pm 6,6 \\
(358-364)\end{array}$ & $\begin{array}{l}316,5 \pm 3 \\
(315-318)\end{array}$ & $\begin{array}{c}360,6 \pm 6,6 \\
(357-362)\end{array}$ & $\begin{array}{l}316,5 \pm 3 \\
(314-317)\end{array}$ \\
\hline 9 & $\begin{array}{r}363,6 \pm 1,4 \\
(362-365)\end{array}$ & $\begin{array}{r}364,2 \pm 3,1 \\
(362-366)\end{array}$ & $\begin{array}{l}348,9 \pm 8 \\
(341-351)\end{array}$ & $\begin{array}{l}283,7 \pm 7 \\
(281-284)\end{array}$ & $\begin{array}{l}348,9 \pm 8 \\
(346-349)\end{array}$ & $\begin{array}{l}283,7 \pm 7 \\
(282-286)\end{array}$ \\
\hline 12 & $\begin{array}{r}368,9 \pm 0,7 \\
(368-370)\end{array}$ & $\begin{array}{l}389 \pm 3,2 \\
(387-391)\end{array}$ & $\begin{array}{c}330,30 \pm 1,4 \\
(328-332)\end{array}$ & $\begin{array}{l}296 \pm 10 \\
(293-301)\end{array}$ & $\begin{array}{c}330,3 \pm 1,4 \\
(330-332)\end{array}$ & $\begin{array}{l}296 \pm 10 \\
(294-301)\end{array}$ \\
\hline 18 & $\begin{array}{r}346,08 \pm 1 \\
(345-348)\end{array}$ & $\begin{array}{c}349,7 \pm 2,8 \\
(346-351)\end{array}$ & $\begin{array}{c}293,19 \pm 1,5 \\
(295-295)\end{array}$ & $\begin{array}{r}260,5 \pm 15 \\
(259-261)\end{array}$ & $\begin{array}{r}293,1 \pm 1,5 \\
(292-294)\end{array}$ & $\begin{array}{r}260,5 \pm 15 \\
(258-265)\end{array}$ \\
\hline 24 & $\begin{array}{c}327,6 \pm 1,6 \\
(326-328)\end{array}$ & $\begin{array}{c}317,8 \pm 9,6 \\
(315-321)\end{array}$ & $\begin{array}{c}268,44 \pm 1,3 \\
(257-269)\end{array}$ & $\begin{array}{c}237 \pm 3 \\
(235-239)\end{array}$ & $\begin{array}{r}268,4 \pm 1,3 \\
(267-268)\end{array}$ & $\begin{array}{c}237 \pm 3 \\
(236-239)\end{array}$ \\
\hline 30 & $\begin{array}{r}317,29 \pm 1 \\
(315-318)\end{array}$ & $\begin{array}{r}281,1 \pm 5,1 \\
(279-283)\end{array}$ & $\begin{array}{c}224,46 \pm 1,5 \\
(222-226)\end{array}$ & $\begin{array}{c}221 \pm 6 \\
(220-223)\end{array}$ & $\begin{array}{r}224,4 \pm 1,5 \\
(223-226)\end{array}$ & $\begin{array}{c}221 \pm 6 \\
(216-222)\end{array}$ \\
\hline 36 & $\begin{array}{c}315 \\
(315-316)\end{array}$ & $\begin{array}{c}252,9 \\
(252-253)\end{array}$ & $\begin{array}{c}197,2 \\
(197-198)\end{array}$ & $\begin{array}{c}199,1 \\
(199-200)\end{array}$ & $\begin{array}{c}201,5 \\
(199-203)\end{array}$ & $\begin{array}{c}199,6 \\
(199-200)\end{array}$ \\
\hline
\end{tabular}

Moy. \pm ET (min-max) : moyenne \pm écart-type (minimum-maximum) // Moy. \pm ET (min-max): mean \pm standard error (minimum-maximum)

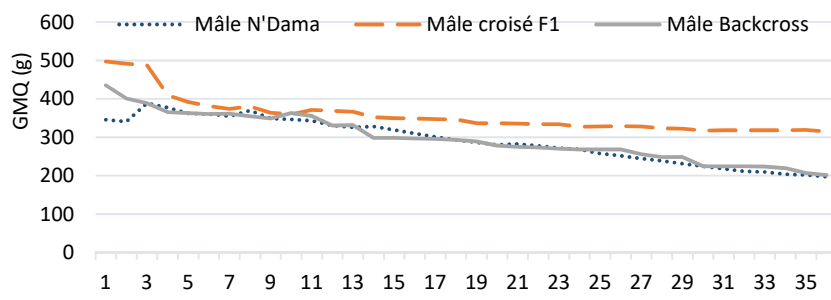

Figure 1 : Evolution du gain de poids moyen quotidien des veaux N'Dama et croisés de la naissance à 36 mois d'âge en Côte d'Ivoire III Average daily weight gain of male N'Dama and crossed calves from birth to 36 months of age in Côte d'Ivoire

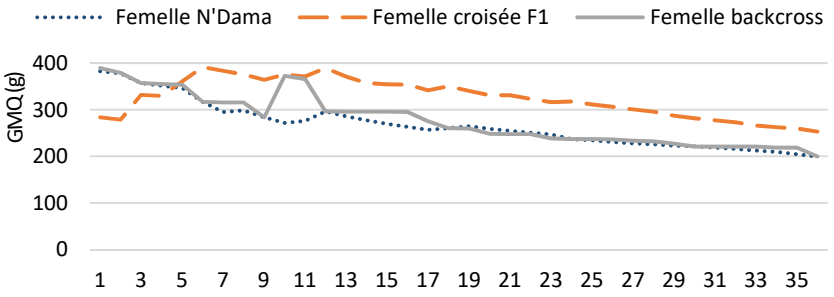

Figure 2 : Evolution du gain de poids moyen quotidien des velles N'Dama et croisées de la naissance à 36 mois d'âge en Côte d'Ivoire I/I Average daily weight gain of female N'Dama and crossed calves from birth to 36 months of age in Côte d'Ivoire

des femelles a favorisé les F1 par rapport aux races pures parentales. En outre, la reproduction des vaches dépendait de la disponibilité de l'inséminateur au moment où les vaches avaient leurs chaleurs. Ces performances sont dues à un non-respect strict des protocoles d'insémination artificielle par les inséminateurs et les éleveurs. Cela se matérialise par une flexibilité sur le choix des vaches à inséminer, le non-respect du calendrier de travail et de la bonne conduite des animaux sélectionnés avant et après l'insémination. Les mêmes constats ont été faits par Benlekhel et al. (2000) au Centre de recherches zootechniques de Minankro, et Sokouri et al. (2010) dans les fermes du Projet laitier Sud en Côte d'Ivoire.
L'âge au premier vêlage des deux niveaux de vaches croisées a été de $38 \pm 3$ mois, alors que celui des vaches N'Dama se situait à $36 \pm 0,5$ mois. La précocité des vaches N'Dama pouvait s'expliquer par les conditions d'élevage où les génisses étaient laissées en permanence en présence des taureaux alors que les demi-sang Montbéliardes étaient incluses dans les programmes d'insémination artificielle. En outre, il y avait un manque de régularité dans l'approvisionnement en produits d'insémination, de même un taureau pouvait ne pas être présent dans le cas de la monte naturelle. Chez les N'Dama la durée moyenne de lactation a été courte (176 \pm 2 jours) comparée à celle des métisses ( $235 \pm 3,3$ jours). La production laitière moyenne par vache N'Dama (1,3 $\pm 0,2 \mathrm{~L})$ a été très faible comparativement aux croisées (7,1 $\pm 0,2 \mathrm{~L})$. Une faiblesse du potentiel laitier des races locales a été relevée. A l'analyse de ces variables, les faibles performances de la N'Dama traduisent la faible productivité de la race (Akouango et al., 2010). En outre, les performances zootechniques des animaux sont influencées par plusieurs facteurs dont les principaux sont l'alimentation, la conduite du troupeau, l'état sanitaire et l'environnement. Dans notre étude, les éleveurs ont effectué des actions sanitaires relatives aux vaccinations, aux déparasitages gastro-intestinaux et externes, afin d'assurer de bonnes conditions de santé aux animaux. Les bons résultats des intervalles entre vêlages $(452 \pm 12,8$ jours et $427,7 \pm 16,6$ jours respectivement chez la N'Dama et la croisée) pouvaient s'expliquer par l'application de programmes de synchronisation des chaleurs et d'insémination artificielle qui ont l'avantage de contrôler et d'induire les chaleurs afin de limiter la période improductive après le vêlage (anœstrus post-partum). Ces intervalles entre vêlages ont été comparables à ceux de 398 × 36,3 jours observés par Ralambofiringa (1978), Coulomb (1980) et Sokouri et al. (2010) chez des vaches croisées.

Le poids à la naissance des N'Dama a été inférieur à celui des croisés de $15 \%$ à $30 \%$. Cela a révélé que le croisement a été améliorateur pour ce paramètre. Les mêmes analyses et observations ont été faites par Landais (1983) et Sokouri et al. (2010) en Côte d'Ivoire. Le poids à âge type des N'Dama a été significativement inférieur à celui des croisés de $12 \%$ à $57 \%(\mathrm{p}<0,05)$. En effet, il a été obtenu à l'âge adulte $370 \mathrm{~kg}$ pour les mâles et $300 \mathrm{~kg}$ pour les femelles chez les 
croisés (demi-sang Montbéliards), contre $235 \mathrm{~kg}$ pour les mâles et $230 \mathrm{~kg}$ pour les femelles N'Dama. Cela s'expliquerait par le fait que le métissage a augmenté les valeurs pondérales des croisés par effet d'hétérosis (Diadhiou, 2002).

Les GMQ ont confirmé que le développement corporel était relativement rapide dans les premiers mois de la vie, notamment de 0 à 3 mois, et diminuait progressivement à mesure que l'âge des animaux augmentait. Ils ont évolué en dents de scie et ont toujours été inférieurs à 500 grammes, comme rapporté par Coulomb (1980) chez la N'Dama. De même Charray et al. (1977) ont constaté chez la N'Dama, en Côte d'Ivoire, un ralentissement perceptible de la vitesse de croissance pendant les quelques mois qui suivaient le sevrage, entre 8 et 11 mois chez le mâle, et 7 et 11 mois chez la femelle. Des observations analogues ont été faites par Poivey et al. (1987) dans les troupeaux sédentaires au nord de la Côte d'Ivoire. Ces auteurs montrent que la vitesse de croissance mensuelle est différente selon l'âge des animaux et maximale de 0 à 3 mois.

\section{CONCLUSION}

La présente étude a montré que les performances de reproduction et de croissance des races croisées (demi-sang Montbéliardes) et N'Dama étaient différentes. Les paramètres de reproduction et de production ont été plus importants chez les croisés que chez la race locale N'Dama. En effet, l'amélioration de ces paramètres serait due à l'effet d'hétérosis qui profite à la production locale ; notamment la production laitière est passée de 1,3 litre/vache/jour chez les N'Dama à 7,1 litres/vache/jour chez les croisées. Ainsi, le croisement de la race exotique Montbéliarde avec la race locale N'Dama a amélioré le potentiel génétique des animaux d'élevage.

\section{Remerciements}

Les auteurs remercient le docteur vétérinaire Ouattara Guy et le Directeur régional Agba Koutouan Mathieu du ministère des Ressources animales et halieutiques ainsi que les deux relecteurs anonymes pour leur contribution.

\section{Déclaration des contributions des auteurs}

L'étude et l'écriture du manuscrit ont été réalisées de façon collégiale. Néanmoins, selon les étapes suivantes, NBA et NS ont participé à la conception et à la planification de l'étude, NBA et MKK ont recueilli les données et rédigé la première version du manuscrit, GKGB et NS ont effectué les analyses statistiques et l'interprétation des données, RYS, MKK et NBA ont effectué la révision critique du manuscrit.

\section{Conflits d'intérêts}

Létude a été réalisée sans conflit d'intérêts.

\section{REFERENCES}

Akaffou N.A., 2013. Elevage des bovins laitiers dans la région du Poro (Côte d'Ivoire) : Situation actuelle et perspectives de développements. Thèse de Doct., Université Cheick Anta Diop de Dakar, Sénégal, 113 p.

Akouango F., Ngokaka C., Ewomango P., Kimbembe E., 2010. Caractérisation morphométrique et reproductive des taureaux et vaches N'Dama du Congo. Anim. Genet. Ressour., 46: 41-47, doi: 10.1017/ S2078633610000688

Atsé A.P., 1990. La production laitière dans le nord de la Côte d'Ivoire. Document SODEPRA Nord, service zootechnie, Korhogo, Côte d'Ivoire, 187 p.

Benlekhel A., Ezzahari A. Bouhaddane A., 2000. L'insémination artificielle des bovins Une biotechnologie au service des éleveurs ». Transf. Technol. Agric., 65: 4

Charray J., Coulomb J., Mathon J.C., 1977. Jersey x N'Dama crossbreeding in Ivory Coast. Performance analysis of half-bred cattle born and bred in Minankro Zootechnical Research Centre. Rev. Elev. Med. Vet. Pays Trop., 30 (1): 67-83, doi: 10.19182/remvt.8107

Coulibaly D., 2013. Politique de développement de l'élevage en Côte d'Ivoire. Direction de la planification et des programmes, Abidjan, Côte d'Ivoire, 31 p.

Coulomb J., 1980. Les races, les modes d'élevages. Premier colloque International: Recherche sur l'élevage bovin en zone tropicale humide, Tome II, Bouaké, Côte d'Ivoire, 543 p.

Diadhiou Y., 2002. Insémination artificielle et production laitière dans le bassin arachidier. Thèse de Doct., Ecole Inter-Etats des Sciences et Médecine Vétérinaires, Dakar, Sénégal, 77 p.

INS, 2014. Recensement général de la population et de l'habitat. Institut national de la statistique, Abidjan, Côte d'Ivoire, 26 p.

Koné A., 2010. Le développement agro-industriel du Nord de la Côte d'Ivoire : cas des départements de Korhogo, Boundiali, Ferkessédougou. Agence Ivoirienne des Sciences Agronomiques, Abidjan, Côte d'Ivoire, 133 p.

Landais E. 1983. Analyse des systèmes d'élevage bovin sédentaire du Nord de la Côte d'Ivoire. Tome 1 et 2. GERDAT-IEMVT, Maisons-Alfort, France, 759 p.

Metzger R., Centres J.P., Lambert J.C., 1995. L'approvisionnement des villes Africaines en lait et en produits laitiers. FAO, Rome, Italie, 124 p.

MIRAH, 2014. Rapport annuel d'activité. Ministère des Ressources animales et Halieutiques, Direction régionale de Man, Côte d'Ivoire, 40 p.

Poivey J.P., Ménissier F., Vissac B., Moussa K., 1987. Growth variability among calves and young bovines from sedentary herds in northern Ivory Coast. Rev. Elev. Med. Vet. Pays Trop., 40 (2): 157-166, doi: 10.19182/remvt.8672

Ralambofiringa A., 1978. Note on the manifestation of the œestrus cycle and on the reproduction in N'Dama cows. Rev. Elev. Med. Vet. Pays Trop., 31 (1): 91-94, doi: 10.19182/remvt.8184

Sokouri D.P., Yapi-Gnaoré C.V., N'guetta A.S.S., Loukou N.E., Kouao B.J. Toure G., Kouassi A. et al., 2010. Performances de reproduction des races bovines locales de la Côte d'Ivoire. J. Appl. Biosci., 36: 2353- 2359 


\section{Summary}

Aboly N.B., Konan M.K., Brou G.K.G., Silué N., Soro R.Y. Reproduction and growth of two breeds of cattle (N'Dama and crossbred) in the Mountains' District of Western Côte d'Ivoire

The study was conducted in the western part of Côte d'Ivoire in the Mountains' District on 120 cows and 96 male and female calves of N'Dama and crossbred (Montbeliard x N'Dama) breeds for 36 months to determine their reproductive and growth performances. The results showed that the pregnancy rate and lactation duration of crossbred cows (half-blood Montbeliard) were higher than those of the N'Dama. Similarly, the calving interval of the crossbred cows ( $428 \pm 16.6$ days) was lower than that of the N'Dama (452 \pm 12.8 days). The birth weight of crossbred calves $(23-25 \mathrm{~kg})$ was higher than that of the N'Dama (17-19 kg). The average daily weight gain over the entire period was 356.8 $\pm 47.7 \mathrm{~g}$ for males and $324.8 \pm 47.1 \mathrm{~g}$ for females in crossbred calves versus $291.7 \pm 57.6 \mathrm{~g}$ and $266.6 \pm 49.1 \mathrm{~g}$, respectively, in N'Dama calves. Finally, the adult weight was $370 \pm 1.7 \mathrm{~kg}$ for males and $300 \pm 2.3 \mathrm{~kg}$ for females in the crossbred group versus $235 \pm 1.6 \mathrm{~kg}$ and $230 \pm 2.1 \mathrm{~kg}$, respectively, in the N'Dama. The reproductive and growth performances of the crossbred males were significantly higher $(p<0.05)$ than those of the N'Dama.

Keywords: cattle, Montbeliard, N'Dama, crossbreds, animal reproduction, animal performance, Côte d'Ivoire

\section{Resumen}

Aboly N.B., Konan M.K., Brou G.K.G., Silué N., Soro R.Y. Reproducción y crecimiento de dos razas de bovinos (N'Dama y cruces) en el distrito de las Montañas al oeste de la Côte d'Ivoire

El estudio se llevó a cabo al oeste de la Côte d'Ivoire en el distrito de las Montañas, en 120 vacas y 96 terneros machos y hembras de razas N'Dama y cruces (Montbéliarde x N'Dama) durante 36 meses con el fin de determinar sus rendimientos de producción y de crecimiento. Los resultados revelaron que la tasa de preñez y la duración de la lactancia de las vacas cruzadas (media sangre Monbéliardes) fueron más elevados que los de las N'Dama. Igualmente, el intervalo entre partos de las vacas cruzadas (428 $\pm 16,6$ días) fue inferior al de las N'Dama (452 $\pm 12,8$ días). El peso al nacimiento de los terneros cruzados (23 a $25 \mathrm{~kg}$ ) fue superior al de los N'Dama (17 a $19 \mathrm{~kg}$ ). La ganancia media cotidiana sobre todo el periodo fue de 356,8 \pm 47,7 g para los machos y $324,8 \pm 47,1$ g para las hembras en los cruzados, contra respectivamente $291,7 \pm 57,6$ g y $266,6 \pm 49,1$ g en los N'Dama. Finalmente, el peso adulto fue de $370 \pm 1,7 \mathrm{~kg}$ para los machos y $300 \pm 2,3 \mathrm{~kg}$ para las hembras en los cruzados, contra respectivamente $235 \pm 1,6 \mathrm{~kg}$ y $230 \pm 2,1 \mathrm{~kg}$ en los N'Dama. Los rendimientos de producción y de crecimiento de los cruzados fueron significativamente más elevados $(p<0,05)$ que los N'Dama.

Palabras clave: ganado bovino, Montbeliard, N'Dama, productos del cruzamiento, reproducción animal, desempeño animal, Côte d'Ivoire 